\title{
Patterns of Motor and Non-Motor Features in Medication-Naïve Parkinsonism
}

\author{
Samay Jain ${ }^{\mathrm{a}}$ Seo-Young Park ${ }^{\mathrm{b}}$ Diane Comer ${ }^{\mathrm{b}}$ \\ ${ }^{a}$ Department of Neurology, UPMC, University of Pittsburgh School of Medicine, ${ }^{b}$ Center for Research on Health Care \\ Data Center, University of Pittsburgh, Pittsburgh, Pa., USA
}

\section{Key Words}

Parkinsonism · Non-motor features · SWEDD $\cdot$ DaTscan

\begin{abstract}
Background: Parkinsonism is defined by motor features (tremor, bradykinesia, rigidity, and postural instability). Accompanying non-motor features (e.g. cognitive, autonomic, sleep disturbances) are underrecognized and undertreated. We hypothesized that clinical patterns occurring in early, medication-naïve Parkinsonism are distinguished by features such as tremor, sleep, autonomic, and cognitive dysfunction. Methods: Clinical and neuroimaging data were obtained in the Parkinson's Progression Marker Initiative. Group comparisons of Parkinsonism with dopaminergic deficits (PDD) $(n=388)$, controls $(n=196)$, and Parkinsonism with scans without evidence of dopaminergic deficits $(n=64)$ were done with ANOVA, chi-square, and post-hoc pairwise tests. To examine clinical patterns within the PDD group, kmeans clustering was performed with non-motor or motor features, or both. Results: Among PDD, 4 non-motor patterns (\% of PDD) (impulsive (14.9\%), sleep-autonomic (22.9\%), cognitive-olfactory (18.0\%), and mild (44.1\%)), 4 motor patterns (tremor plus bradykinesia $(56.2 \%)$, tremor without bradykinesia (16.2\%), postural instability (6.7\%) and no tremor (20.9\%)) and 5 combined motor/non-motor patterns (tremor with bradykinesia (42.3\%), tremor without bra-
\end{abstract}

dykinesia (15.5\%), no tremor and mild non-motor features (17.0\%), postural instability with sleep-autonomic disturbances (6.7\%) and oldest onset cognitive-olfactory (18.6\%)) were observed. Conclusions: To our knowledge, this is the first description of non-motor clinical patterns in early, medication-naïve Parkinsonism, suggesting that such features are intrinsic to Parkinsonian disorders. @ $2015 \mathrm{~S}$. Karger AG, Basel

\section{Introduction}

Parkinsonism is common in neurological outpatient clinics, with an incidence ranging from $0.5 / 1,000$ personyears between the ages of 55 and 65 years, to $10.6 / 1,000$ for those over 85 years of age [1]. As there is currently no diagnostic test for Parkinsonism, identification relies solely on clinical signs comprised of disturbances in characteristic motor features (e.g. tremor, bradykinesia, rigidity, gait instability), which are also the focus of treatment. Clinical and pathological evidence suggests that several idiopathic Parkinsonian disorders are multi-system, multi-organ diseases in which motor deficits are accompanied by non-motor features including cognitive, autonomic, psychiatric, and sleep disturbances [2]. Non-motor features in Parkinson's disease predate motor dysfunction by more than 20 years, and are linked to widespread

\section{KARGER 125}

(c) 2015 S. Karger AG, Base

$0251-5350 / 15 / 0451-0059 \$ 39.50 / 0$ 
Table 1. Tests performed on all participants in PPMI

\begin{tabular}{ll}
\hline Clinical assessments & \\
\hline Motor (higher score is worse) & $\begin{array}{l}\text { Movement Disorders Society sponsored Unified Parkinson disease } \\
\text { Rating Scale (MDS-UPDRS) }\end{array}$ \\
\hline $\begin{array}{ll}\text { Neurobehavior } \\
\text { (higher scores are worse) }\end{array}$ & $\begin{array}{l}\text { Geriatric depression scale } \\
\text { State-trait anxiety inventory } \\
\text { Questionnaire for impulsive-compulsive disorders } \\
\text { (screening: any 1 item is considered positive) }\end{array}$ \\
\hline $\begin{array}{ll}\text { Cognitive testing } \\
\text { (lower scores are worse) }\end{array}$ & $\begin{array}{l}\text { Montreal Cognitive Assessment } \\
\text { Hopkins verbal learning test - revised } \\
\text { Benton judgment of line orientation }\end{array}$ \\
& $\begin{array}{l}\text { Semantic fluency } \\
\text { Letter number sequencing } \\
\text { Symbol digit modalities test }\end{array}$ \\
\hline $\begin{array}{l}\text { Autonomic symptoms } \\
\text { (higher score is worse) }\end{array}$ & Scales for Outcomes in Parkinson's disease - Autonomic (SCOPA-AUT) \\
\hline $\begin{array}{l}\text { Sleep disorders } \\
\text { (higher scores are worse) }\end{array}$ & $\begin{array}{l}\text { Epworth sleepiness scale } \\
\text { REM sleep disorder questionnaire }\end{array}$ \\
\hline $\begin{array}{l}\text { Olfactory testing } \\
\text { (lower score is worse) }\end{array}$ & University of Pennsylvania Smell Identification Test (UPSIT) \\
\hline $\begin{array}{l}\text { Imaging and biospecimen collection } \\
\text { Dopamine transporter imaging }\end{array}$ & [ ${ }^{123}$ I]-FP-CIT single photon emission computerized tomography (DaTscan) \\
\hline
\end{tabular}

neuropathological changes throughout the nervous system [3]. Although non-motor features often have greater impact on healthcare costs, quality of life, and institutionalization rates than motor features, they are underrecognized $[4,5]$. Yet several non-motor features are treatable.

We used data collected by the Parkinson's Progression Marker Initiative (PPMI) to test our hypothesis that specific patterns of non-motor features occur in patients with early idiopathic Parkinsonism who have never received anti-Parkinsonian medication. We sought to characterize clinical patterns of early Parkinsonism integrating non-motor and motor features because recognizing these patterns early in the course of Parkinsonism would facilitate development of more comprehensive early treatment strategies and inform concepts of pathogenesis. As deficits in dopamine neurotransmission underlie most idiopathic Parkinsonism, we also examined dopaminergic deficits in participants using data collected from neuroimaging, which labeled dopamine transporters in the striatum. The status of striatal dopamine transporters led to 2 main groups of Parkinsonism: Parkinsonism with scans without evidence of dopaminergic deficits (SWEDD's) and those with Parkinsonism with dopaminergic deficits (PDD).

\section{Materials and Methods}

\section{Study Population}

The PPMI is an ongoing observational multicenter cohort study designed to identify Parkinson disease progression markers comprised of three groups: PDD $(n=388)$, controls $(n=196)$, and SWEDD's $(\mathrm{n}=64)$. Details are published elsewhere and available at http://www.ppmi-info.org/ [6]. The study was approved by the institutional review board of all participating sites. Written informed consent was obtained from all participants. Healthy control subjects had no significant neurological dysfunction, no first-degree relative with Parkinson disease, a Montreal Cognitive Assessment $>26$, and no detectable dopamine transporter deficit on neuroimaging (DaTscan, methods detailed later in this article). Participants with Parkinsonism were diagnosed less than 2 years prior to the screening visit, untreated, and required to have an asymmetric resting tremor or asymmetric bradykinesia or 2 of bradykinesia, resting tremor and rigidity. Patients with clinical findings consistent with early stage Parkinsonism, no history of secondary causes of Parkinsonism and a dopamine transporter deficit on DaTscan imaging constituted the PDD group, and those without evidence of dopaminergic deficit comprised the SWEDD group. All participants underwent tests summarized in table 1 . We used data from the earliest time available (screening, baseline or first follow-up visit) downloaded from the PPMI database (download dates were July 9, 2013 for clinical assessments and September 10, 2013 for imaging).
60
Jain/Park/Comer 


\section{Assessment of Clinical Features}

Demographic and historical information on all participants were obtained including age, sex, years of education, age of onset of first symptoms of Parkinson's disease, motor features present at diagnosis, and date of diagnosis. Parkinsonism was assessed by the Movement Disorder Society sponsored Unified Parkinson Disease Rating Scale (MDS-UPDRS). Similar to other studies [7, 8], motor features were summarized by taking the average score of MDS-UPDRS individual items for postural instability/gait (sum of items (3.10 through 3.14)/4), hypokinesia/rigidity (sum of items (3.1 through $3.9+3.14) / 9)$, and tremor (sum of items (3.15 through $3.18) / 10)$. Non-motor features were measured by the sum of MDS-UPDRS Part 1 and specific assessments of olfaction, cognition, sleep, autonomic, and psychiatric features are summarized in table 1.

\section{Neuroimaging}

All subjects underwent dopamine transporter imaging by DaTscan (an intravenous injection of $\left[{ }^{123} \mathrm{I}\right]$-FP-CIT containing activity in the range of 111-185 MBq over 15-20 s followed by single photon emission computerized tomography imaging done 3-6 h later) [9]. Details are available on the PPMI website at http://www. ppmi-info.org/. Regions of interest were placed on the left and right caudate, the left and right putamen, and the occipital cortex (reference tissue). Count densities for each region were used to calculate striatal binding ratios (SBRs) for each of the four striatal regions $(\mathrm{SBR}=($ target region $/$ reference region $)-1)$.

\section{Statistical Analyses}

Descriptive statistics, ANOVA $F$ tests, $t$ tests, and chi-square tests summarized baseline characteristics and group comparisons. Three k-means cluster analyses were performed among PDD participants: one based on 14 non-motor variables, 1 based on 7 motor variables, and 1 based on both non-motor and motor variables. We included 388 of the 423 PDD participants without any missing values for clustering. Variables were standardized before clustering so that each had a mean 0 and standard deviation 1 . For binary variables, we assigned values 0 or 1 and treated them in the same way as continuous variables. As we standardized all variables before clustering so that each variable has mean 0 and standard deviation 1 , these values ( 0 and 1$)$ might have changed. To empirically determine the number of clusters, we compared the sum of squared error (SSE) for a number of cluster solutions [10]. SSE is the sum of the squared distance between each member of a cluster and its cluster centroid. We looked for a point with a sudden drop of SSE to find the number of clusters. Also, we produced 250 randomized versions of the original input data by randomly scrambling all entries of the data matrix, and calculated SSE against cluster solutions for the randomized data. If a data set has strong clusters, the SSE of the actual data should decrease more quickly than the random data as the number of clusters increase. We also looked at the Gap statistic as another measure for estimating the number of clusters [11]. In this way, we chose 4,4 , and 5 clusters for clustering based on non-motor, motor, and combined variables, respectively. We then compared the resulting clusters with ANOVA F test and chi-square test for continuous and binary variables, respectively. For variables that were significantly different across clusters ( $\mathrm{p} \leq 0.05)$, we performed post-hoc pairwise analysis using ANOVA with a Tukey adjustment. We labeled clinical patterns using descriptors based on variables that were significantly different among clusters. For example, if cognitive and olfactory testing were significantly worse in a particular cluster, that cluster was labeled cognitive-olfactory (SAS version 9.3 (2012) [12] was used to prepare downloaded datasets then analyzed by R version 3.0.1 (2013) [13]).

\section{Results}

Group comparisons are in table 2. Compared to controls, PDD dopamine transporter imaging SBRs and olfactory function (University of Pennsylvania Smell Inventory (UPSIT) scores) were lower and scores for posture/gait, scores for hypokinesia/rigidity and tremor abnormalities were higher. SWEDDs scored the highest in severity of non-motor features for the MDS-UPDRS Part 1, Scale for Outcome of Parkinson Disease - Autonomic (SCOPA-AUT), and Epworth Sleepiness Scale and had the highest proportion of individuals with impulsive/ compulsive behaviors. Controls performed best in most cognitive tests.

Clustering using non-motor features yielded four patterns in the PDD group (table 3): (1) Impulsive: presence of impulsive/compulsive behaviors; (2) Sleep-autonomic: most severe non-motor (MDS-UPDRS Part 1), autonomic (SCOPA-AUT) and REM sleep disorder symptoms; (3) Cognitive-olfactory: performed worst on all cognitive tests and had low UPSIT scores; and (4) Mild: no impulsive/compulsive behaviors and the best UPSIT performance. This 4 cluster solution accounted for $24.7 \%$ of the variance.

Clustering using motor features also yielded 4 patterns in the PDD group (table 4): (1) Tremor plus bradykinesia: tremor and bradykinesia at the time of diagnosis; (2) Tremor without bradykinesia: tremor and no bradykinesia at the time of diagnosis; (3) Postural instability: postural instability at the time of diagnosis and the highest posture/gait scores at baseline; and (4) No tremor: no tremor at the time of diagnosis. This 4 cluster solution accounted for $47.0 \%$ of the variance.

When we used both non-motor and motor features for clustering, 5 patterns emerged in the PDD group (table 5): (1) tremor plus bradykinesia and (2) tremor without bradykinesia were characterized as described earlier; (3) no tremor and mild non-motor symptoms (no tremor-mild): no tremor at diagnosis and lower severity for several non-motor features; (4) postural instability with sleep and autonomic features: postural instability at the time of diagnosis and the most severe sleep and autonomic symptoms; and (5) oldest onset cognitive-olfactory: the 
Table 2. Group comparisons

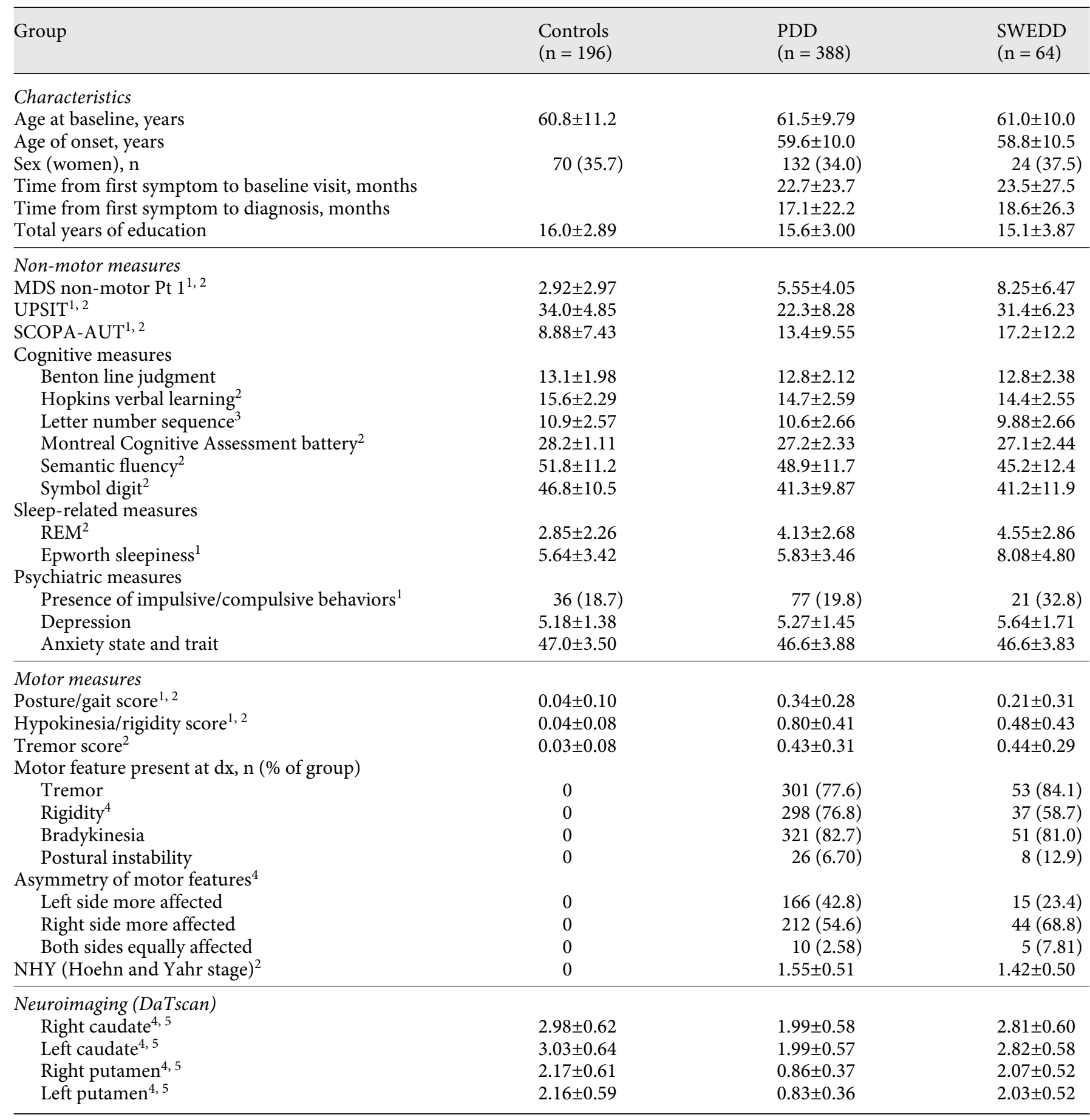

Results are given as raw scores $\pm \mathrm{SD}$, values in parentheses are percentages.

${ }^{1}$ SWEDD group is significantly different from control and PDD groups.

2 Control group is significantly different from SWEDD and PDD groups.
${ }^{3}$ SWEDD group is significantly different from control group but not from PDD group.

${ }^{4} \mathrm{PDD}$ group is significantly different from SWEDD group but not controls.

${ }^{5}$ Control group is significantly different from PDD group but not SWEDD group. 
Table 3. Results of clustering using only non-motor features

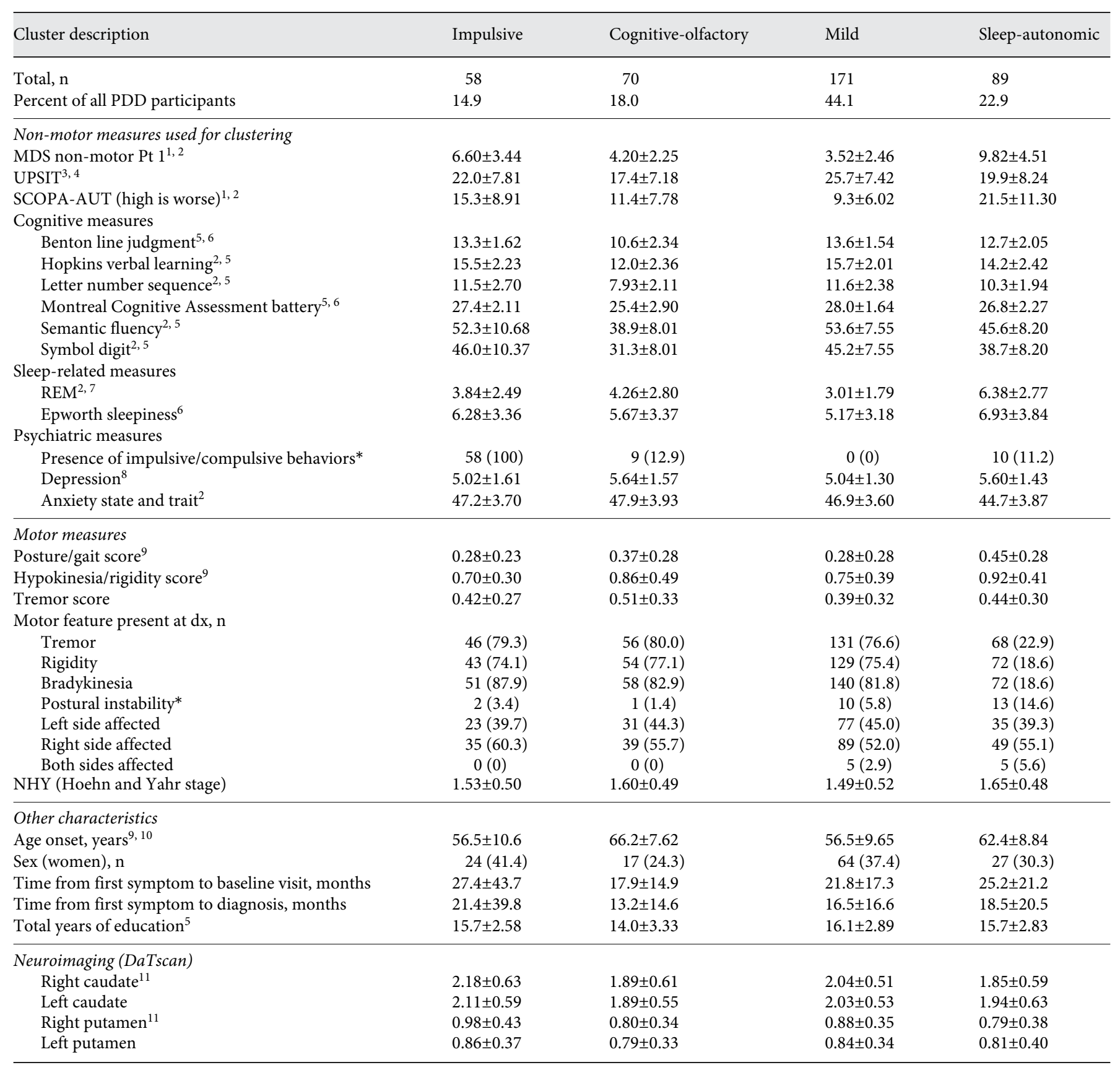

Results are given as raw scores \pm SD, values in parentheses are percentages of cluster, and significant differences are $\mathrm{p}<0.05$ after Tukey adjustment. ${ }^{*} \mathrm{p}<0.05$ : significantly different by chi-square.

${ }^{1}$ Impulsive group significantly different from all other groups.

${ }^{2}$ Sleep/autonomic group significantly different from all other groups.

${ }^{3}$ Mild significantly different from all other groups.

${ }^{4}$ Impulsive group significantly different from olfactory/cognition group.

${ }^{5}$ Cognitive-olfactory group significantly different from all other groups.
${ }^{6}$ Sleep/autonomic group significantly different from mild group.

${ }^{7}$ Mild group significantly different from cognitive-olfactory group.

${ }^{8}$ Mild group significantly different from sleep/autonomic and cognitive-olfactory group.

${ }^{9}$ Sleep/autonomic group significantly different from impulsive and mild groups.

${ }^{10}$ Cognitive-olfactory group significantly different from impulsive and mild groups.

${ }^{11}$ Impulsive group significantly different from sleep/autonomic and cognitive-olfactory group.
Clinical Patterns in Medication-Naïve Parkinsonism 
Table 4. Results of clustering using only motor features

\begin{tabular}{|c|c|c|c|c|}
\hline Cluster description & $\begin{array}{l}\text { Tremor with } \\
\text { bradykinesia }\end{array}$ & Tremor-no-bradykinesia & Postural instability & No tremor \\
\hline Total $(\mathrm{n}=388)$ & 218 & 63 & 26 & 81 \\
\hline \multicolumn{5}{|l|}{ Motor characteristics used for clustering } \\
\hline \multicolumn{5}{|l|}{ Motor feature present at dx, n } \\
\hline Bradykinesia* & $218(100)$ & $0(0)$ & $24(92.3)$ & $79(97.5)$ \\
\hline Postural instability* & $0(0)$ & $0(0)$ & $26(100)$ & $0(0)$ \\
\hline Posture/gait score ${ }^{1}$ & $0.32 \pm 0.27$ & $0.29 \pm 0.27$ & $0.55 \pm 0.34$ & $0.34 \pm 0.29$ \\
\hline Hypokinesia/rigidity score ${ }^{2}$ & $0.80 \pm 0.39$ & $0.72 \pm 0.43$ & $0.98 \pm 0.47$ & $0.82 \pm 0.40$ \\
\hline Tremor score s $^{3}$ & $0.49 \pm 0.31$ & $0.58 \pm 0.28$ & $0.45 \pm 0.22$ & $0.15 \pm 0.17$ \\
\hline \multicolumn{5}{|l|}{ Cognitive measures } \\
\hline Benton line judgment & $12.8 \pm 2.11$ & $12.4 \pm 2.32$ & $13.0 \pm 1.80$ & $12.9 \pm 2.11$ \\
\hline Hopkins verbal learning & $14.7 \pm 2.60$ & $14.6 \pm 2.58$ & $14.3 \pm 2.59$ & $14.7 \pm 2.63$ \\
\hline Letter number sequence & $10.6 \pm 2.64$ & $10.5 \pm 2.68$ & $10.4 \pm 2.63$ & $10.8 \pm 2.75$ \\
\hline Montreal Cognitive Assessment battery ${ }^{2}$ & $27.2 \pm 2.42$ & $26.6 \pm 2.39$ & $28.0 \pm 1.62$ & $27.4 \pm 2.13$ \\
\hline Semantic fluency & $49.0 \pm 11.3$ & $48.2 \pm 11.9$ & $49.2 \pm 10.5$ & $49.0 \pm 13.2$ \\
\hline Symbol digit & $40.6 \pm 10.26$ & $43.0 \pm 8.51$ & $41.2 \pm 8.57$ & $42.1 \pm 10.1$ \\
\hline \multicolumn{5}{|l|}{ Sleep-related measures } \\
\hline REM $^{1}$ & $4.12 \pm 2.61$ & $3.76 \pm 2.35$ & $5.57 \pm 3.37$ & $3.99 \pm 2.76$ \\
\hline Epworth sleepiness & $5.70 \pm 3.38$ & $5.51 \pm 3.61$ & $7.54 \pm 3.57$ & $5.89 \pm 3.44$ \\
\hline \multicolumn{5}{|l|}{ Psychiatric measures } \\
\hline Presence of impulsive/compulsive behaviors & $48(22.0)$ & $9(14.3)$ & $7(26.9)$ & $13(16.0)$ \\
\hline Time from first symptom to diagnosis, months & $18.3 \pm 25.8$ & $15.6 \pm 16.9$ & $21.5 \pm 23.2$ & $13.8 \pm 13.3$ \\
\hline \multicolumn{5}{|l|}{ Side affected at time of diagnosis } \\
\hline Left side affected at diagnosis & $97(44.5)$ & $19(30.2)$ & $12(46.2)$ & $38(46.9)$ \\
\hline Right side affected at diagnosis & $117(53.7)$ & $43(68.3)$ & $12(46.2)$ & $40(49.4)$ \\
\hline Both sides affected at diagnosis & $4(1.8)$ & $1(1.6)$ & $2(7.7)$ & $3(3.8)$ \\
\hline Total years of education & $15.6 \pm 3.02$ & $15.7 \pm 3.00$ & $16.1 \pm 2.32$ & $15.0 \pm 3.13$ \\
\hline \multicolumn{5}{|l|}{ Neuroimaging (DaTscan) } \\
\hline Right caudate & $1.99 \pm 0.54$ & $2.11 \pm 0.58$ & $1.87 \pm 0.65$ & $1.93 \pm 0.61$ \\
\hline Left caudate & $2.01 \pm 0.55$ & $2.14 \pm 0.59$ & $1.94 \pm 0.60$ & $1.88 \pm 0.57$ \\
\hline Right putamen & $0.86 \pm 0.39$ & $0.96 \pm 0.36$ & $0.76 \pm 0.34$ & $0.82 \pm 0.31$ \\
\hline Left putamen & $0.85 \pm 0.38$ & $0.85 \pm 0.32$ & $0.74 \pm 0.22$ & $0.78 \pm 0.35$ \\
\hline \multicolumn{2}{|c|}{$\begin{array}{l}\text { Results are given as raw scores } \pm \mathrm{SD} \text {, values in parentheses are } \\
\text { percentages of cluster, and significant differences are } \mathrm{p}<0.05 \text { af- } \\
\text { ter Tukey adjustment. }{ }^{*} \mathrm{p}<0.05 \text { : significantly different by chi- } \\
\text { square. } \\
\quad 1 \text { Postural instability significantly different from all other } \\
\text { groups. }\end{array}$} & \multicolumn{3}{|c|}{$\begin{array}{l}{ }^{2} \text { Postural instability significantly different from tremor-no- } \\
\text { bradykinesia. } \\
{ }^{3} \text { No tremor significantly different from all other groups. } \\
{ }^{4} \text { Postural instability significantly different from tremor-no- } \\
\text { bradykinesia and no-tremor groups. } \\
{ }^{5} \text { No tremor significantly different from tremor-no-bradykinesia. }\end{array}$} \\
\hline
\end{tabular}




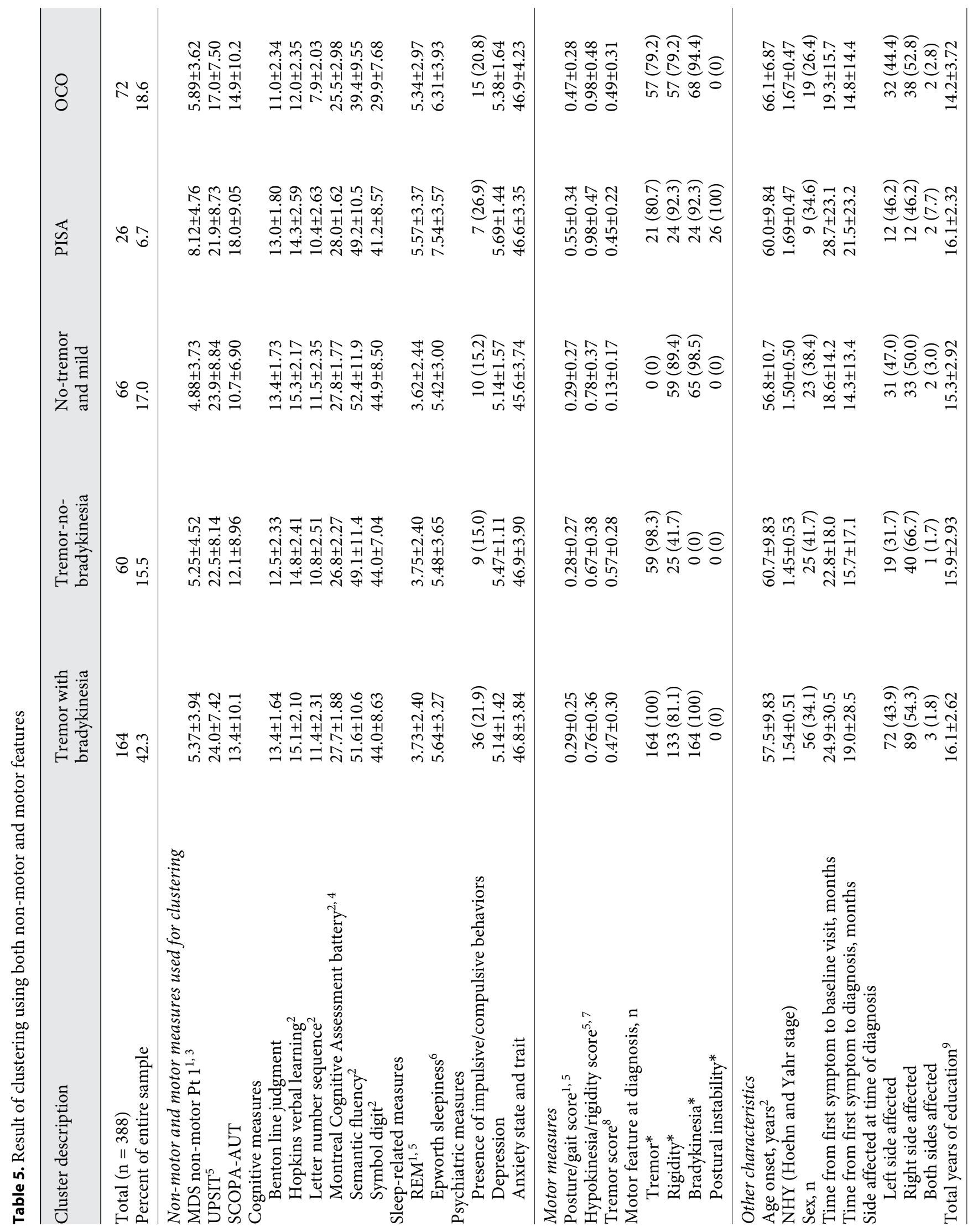




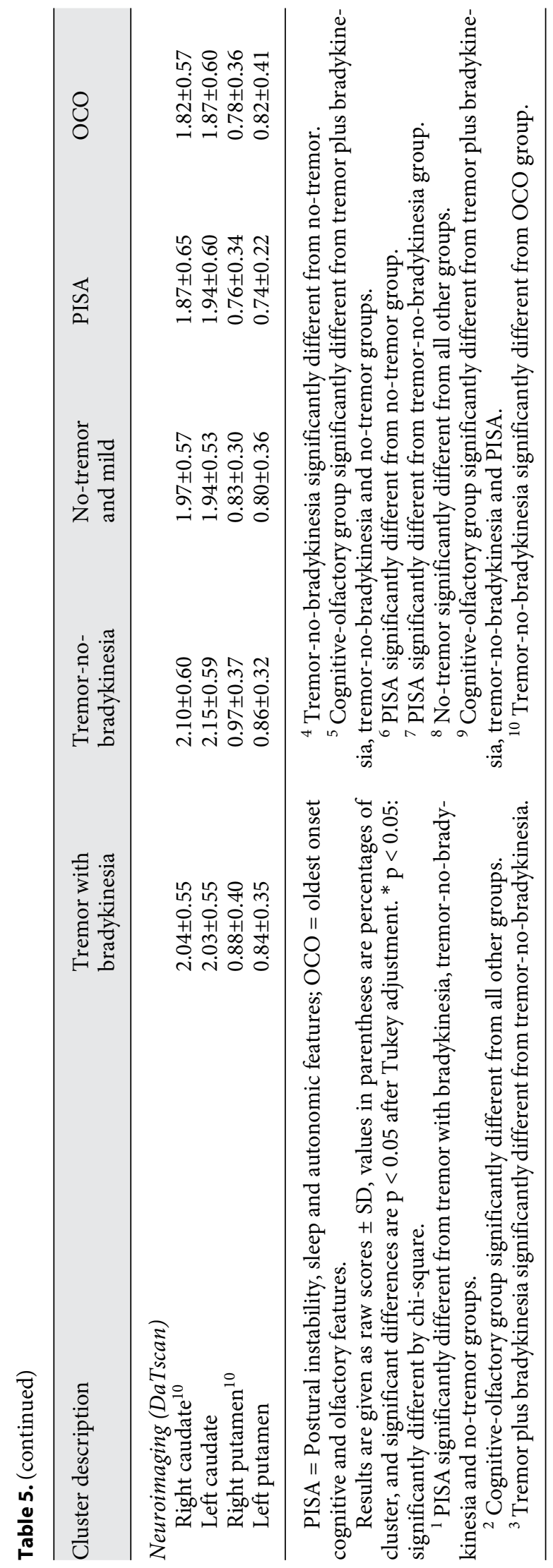

oldest age of onset of PDD with the worst cognitive and olfactory performance. This 5 cluster solution accounted for $22.7 \%$ of the variance.

\section{Discussion}

To our knowledge, this is the first report characterizing non-motor clinical patterns in early medication naïve Parkinsonism. We found non-motor symptoms, particularly sleep and autonomic features, to be worse in SWEDDs than in controls or PDD. We also found SWEDDs to have the highest prevalence of impulsive/ compulsive behaviors, and this may result in them reporting more non-motor symptoms. Although SWEDDs could be very early Parkinsonism, which later demonstrate dopamine transport deficits, according to other studies follow-up of SWEDDs over 4 years does not demonstrate decreasing striatal dopamine in most cases [1416]. SWEDDs may represent other conditions such as secondary Parkinsonism, Huntington disease, adult-onset dystonic tremor, essential tremor, psychogenic tremor or Fragile X Permutation [17]. As in another study, we found olfactory function in SWEDDs was better than in Parkinson's disease [18]. Unlike our results, a separate study reported that SWEDDs have less severe non-motor issues of urinary symptoms, sleep disturbances, and behavior as reported by the Non-Motor Symptoms Scale [19]. This study adds to prior reports because we focus on clinical patterns in early medication naïve Parkinsonism with confirmed status of striatal dopamine transporter binding. Unlike our study, others employed clustering using non-motor features did so in Parkinson disease after a substantial proportion of participants were exposed to dopaminergic treatment, when the controls were not included, and when the means to distinguish SWEDDs was not available [20,21]. Others have investigated clusters later in the disease course $[22,23]$. A major concern about non-motor features is the degree to which they are intrinsic to Parkinsonism, as some may be secondary to medication side effects. For example, dopaminergic medication may contribute to impulse control disorders (ICDs), psychosis, or orthostatic hypotension [24]. Our results about participants who have never exposed to anti-Parkinsonian medication provide strong evidence that such non-motor features are also intrinsic to Parkinsonism.

Regarding non-motor patterns, the impulsive pattern includes ICDs such as gambling, shopping, sexual behavior, and eating [25]. Younger age of onset of Parkinson 
disease is associated with ICDs [26], and this cluster was younger than the sleep-autonomic and cognitive-olfactory patterns. Dopamine is involved in regulating motivation, drive and learning stimulus-reinforcement behaviors [27]. The impulsive pattern had significantly higher dopamine transporter binding in the right caudate and putamen than the sleep-autonomic and cognitive-olfactory patterns. SWEDDs, which were excluded from clustering analyses, had higher dopamine transporter binding and also had higher impulsivity scores.

The sleep-autonomic pattern is consistent with studies finding autonomic dysfunction among those with REM sleep behavior disorder (RBD) $[28,29]$. RBD is a parasomnia in which patients 'act-out' dreams with motor movements while in REM sleep. Neurodegenerative diseases eventually develop in up to $80 \%$ of RBD cases, with the most common being Parkinson's disease [30]. The cognitive-olfactory pattern is consistent with olfactory test scores showing correlations with verbal memory and executive performance [31]. The duration of education for the cognitive-olfactory pattern was lowest among all clusters. This supports the concept of cognitive reserve, which posits that lifelong experiences, including education, can increase cognitive tolerance of age- or diseaserelated changes [32]. The mild non-motor pattern may reflect non-motor features in their nascent phase that may become apparent as Parkinsonism progresses, or a milder non-motor variant of Parkinsonism.

Clustering patterns that we observed with motor features of tremor-predominant and postural-instability/ gait subtypes $[7,33]$ are similar to those observed in other reports about Parkinson's disease. The postural instability pattern had the most severe non-motor features. To our knowledge, this is the first report of 2 motor patterns: tremor with bradykinesia and tremor without bradykinesia.

Although the numeric differences in individual variables among the clusters are small, the usefulness of this study lies in the combination of variables resulting in distinct clinical patterns. These results suggest that specific patterns of non-motor features manifest early in the course of Parkinsonism. However, this is a single analysis based on data obtained at an early point in disease. Before the clinical significance of these patterns can be established, the evolution of these clinical patterns with longitudinal follow-up is necessary. Within PDD, DaTscan results were similar despite the clinically heterogeneous patterns observed. It is possible that either non-dopaminergic pathways or areas outside of the striatum underlie non-motor or motor features.

Clinical Patterns in Medication-Naïve Parkinsonism
The pathological hallmark of the most common form of PDD, Parkinson's disease, is Lewy-related pathology. Lewy formations are aggregates of the protein $\alpha$-synuclein, and their distribution throughout the nervous system is thought to underlie both motor and non-motor features. Recently, Lewy-related pathology has been recognized to occur throughout the brain, spinal cord, and peripheral autonomic nervous system in Parkinson's disease, and these regions underlie non-motor features. Neuropathological variability in extranigral regions may account for the clinical heterogeneity that we observed. For example, the density of $a$-synculein pathology in the olfactory bulb corresponds with olfactory deficits and correlates significantly with cognitive testing (the mini-mental state exam), supporting our clinical pattern of OCO. Multiple pathological studies demonstrate that non-tremor Parkinson disease cases have more severe cortical Lewy pathology, and clinically these cases are more likely to have some cognitive impairment [34].

Although medication-naïve Parkinsonism allows one to appreciate clinical features that are not secondary to medication effects, a major limitation is that we are limited in our ability to diagnose specific Parkinsonian syndromes. This is due to the fact that diagnostic criteria for Parkinson's disease, the most common PDD, include excellent response to dopaminergic medication, and lack of response may lead one to suspect another Parkinsonian disorder. While we were able to determine the status of striatal dopaminergic transporters with DaTscan, in the absence of a trial of dopaminergic medication, it is possible a clinical pattern in PDD could, in part, represent another disorder, such as multiple system atrophy in which autonomic dysfunction is more prominent. Results of cluster analysis are dependent on the variables selected for clustering, such that if different researchers used different features, different clinical patterns may emerge. Replication in other cohorts is necessary to determine validity and generalizability. Given that the PPMI includes those with very early Parkinsonism, it is possible that long-term clinical patterns have not yet emerged, particularly among mild non-motor patterns.

\section{Conclusions}

Although the temporal sequence of non-motor and motor features cannot be determined in our cross-sectional analyses, this study suggests that heterogeneity in Parkinsonism exists very early in the course of disease. The presence of non-motor features in medication-naïve

Neuroepidemiology 2015;45:59-69 DOI: $10.1159 / 000437228$ 
participants suggests that non-motor features are intrinsic to Parkinsonian disorders. Even within the first 3 years of diagnosis among untreated Parkinson disease patients, non-motor symptoms make a larger contribution to diminished quality of life compared to motor features [5]. Several non-motor symptoms are treatable, including sleep disturbances, autonomic dysfunction, cognitive impairment, and psychiatric disorders. The presence of non-motor patterns in early Parkinsonism demonstrates the need for comprehensive treatment strategies, which encompass both motor and non-motor features to begin near the time of diagnosis.

\section{Acknowledgments and Funding}

Data used in the preparation of this article were obtained from the PPMI database (www.ppmi-info.org/data). For up-to-date information on the study, visit www.ppmi-info.org. This project was funded by the Michael J. Fox Foundation for Parkinson's Research and the NIH/NINDS 1 K23 NS070867. The PPMI - a public-private partnership - is funded by the Michael J. Fox Foundation for Parkinson's Research and funding partners, including Biogen Idec, F. Hoffman-La Roche Ltd., GE Healthcare, Genentech, Ab- bvie, Avid Radiopharmaceuticals, Bristol-Myers Squibb, Covance, GlaxoSmithKline, Lilly, Lundbeck, Merk, Meso Scale Discovery, Piramal, UCB and Pfizer Inc.

\section{Disclosure Statement} close.

The authors declare that they have no conflict of interest to dis-

\section{Author Roles}

S. Jain: conception, organization, execution of study design, wrote first draft and final draft of manuscript. S.-Y. Park: statistical analyses - design, execution, review and critique of methods and manuscript. D. Comer: statistical analyses - dataset preparation and design for statistical analyses. Data access and responsibility: S. Jain takes responsibility for the integrity of the data and the accuracy of the data analysis.

\section{Financial Disclosures}

None.

\section{References}

-1 Aerts MB, Esselink RA, Post B, van de Warrenburg BP, Bloem BR: Improving the diagnostic accuracy in Parkinsonism: a three-pronged approach. Pract Neurol 2012;12:77-87.

2 Jellinger KA: Formation and development of Lewy pathology: a critical update. J Neurol 2009;256(suppl 3):270-279.

-3 Jellinger KA: Neuropathobiology of non-motor symptoms in Parkinson disease. J Neural Transm 2015, Epub ahead of print.

4 Chaudhuri KR, Healy DG, Schapira AH: Non-motor symptoms of Parkinson's disease: diagnosis and management. Lancet Neurol 2006;5:235-245.

5 Müller B, Assmus J, Herlofson K, Larsen JP, Tysnes OB: Importance of motor vs. non-motor symptoms for health-related quality of life in early Parkinson's disease. Parkinsonism Relat Disord 2013;19:1027-1032.

6 Parkinson Progression Marker Initiative: The Parkinson progression marker initiative (PPMI). Prog Neurobiol 2011;95:629-635.

7 Lewis SJ, Foltynie T, Blackwell AD, Robbins TW, Owen AM, Barker RA: Heterogeneity of Parkinson's disease in the early clinical stages using a data driven approach. J Neurol Neurosurg Psychiatry 2005;76:343-348.

8 Zetusky WJ, Jankovic J, Pirozzolo FJ: The heterogeneity of Parkinson's disease: clinical and prognostic implications. Neurology 1985;35: 522-526.
-9 Benamer TS, Patterson J, Grosset DG, Booij J, de Bruin K, van Royen E, et al: Accurate differentiation of Parkinsonism and essential tremor using visual assessment of [123I]-FPCIT SPECT imaging: the [123I]-FP-CIT study group. Mov Disord 2000;15:503-510.

10 Peeples M: R Script for K-Means Cluster Analysis. 2011. http://wwwmattpeeplesnet/ kmeanshtml (accessed January 7, 2015).

11 Tibshirani R, Walther G, Hastie T: Estimating the number of clusters in a dataset via the gap statistic. J R Stat Soc Series B 2000;63:411423.

12 What's New in SAS 9.3. Cary, NC, SAS Institute, Inc., 2012

13 Team RC: R: A language and environment for statistical computing Vienna, Austria, R Foundation for Statistical Computing, 2013.

14 Marek K, Jennings D, Seibyl J, et al: Longterm follow-up of patients with scans without evidence of dopaminergic deficit (SWEDD) in the ELLDOPA study. Neurology 2005; 64(suppl 1):A274.

15 Peall K, Morris H, Bajaj N: SWEDD-UK meeting report. Adv Clin Neurosci Rehabil 2010;10:32-37.

-16 Batla A, Erro R, Stamelou M, Schneider SA, Schwingenschuh P, Ganos C, et al: Patients with scans without evidence of dopaminergic deficit: a long-term follow-up study. Mov Disord 2014;29:1820-1825.
17 Bajaj N: SWEDD for the general neurologist. Adv Clin Neurosci Rehabil 2010;10:30-31.

18 Silveira-Moriyama L, Schwingenschuh P, O’Donnell A, Schneider SA, Mir P, Carrillo F, et al: Olfaction in patients with suspected parkinsonism and scans without evidence of dopaminergic deficit (SWEDDs). J Neurol Neurosurg Psychiatry 2009;80:744-748.

19 Jang WAJ, Kim HT: Non-motor symptoms in PD patients with SWEDDs (abstract). Mov Disord 2013;28(suppl 1):299.

20 Erro R, Vitale C, Amboni M, Picillo M, Moccia $\mathrm{M}$, Longo $\mathrm{K}$, et al: The heterogeneity of early Parkinson's disease: a cluster analysis on newly diagnosed untreated patients. PLoS One 2013;8:e70244.

21 Yang HJ, Kim YE, Yun JY, Kim HJ, Jeon BS: Identifying the clusters within nonmotor manifestations in early Parkinson's disease by using unsupervised cluster analysis. PLOS One 2014;9:e91906.

22 van Rooden SM, Colas F, Martínez-Martín P, Visser M, Verbaan D, Marinus J, et al: Clinical subtypes of Parkinson's disease. Mov Disord 2011;26:51-58

23 Thenganatt MA, Jankovic J: Parkinson disease subtypes. JAMA Neurol 2014;71:499504

24 Connolly BS, Lang AE: Pharmacological treatment of Parkinson disease: a review. JAMA 2014;311:1670-1683. 
25 Weintraub D: Impulse control disorders in Parkinson's disease: prevalence and possible risk factors. Parkinsonism Relat Disord 2009; 15(suppl 3):S110-S113.

26 Weintraub D, Nirenberg MJ: Impulse control and related disorders in Parkinson's disease. Neurodegener Dis 2013;11:63-71.

27 Jain S, Waters CH: Controversies of dopamine agonists: somnolence, cardiac valvulopathy and repetitive behaviors. Current Drug Therapy 2007;2:17-20.

28 Postuma RB, Gagnon JF, Montplaisir JY: REM sleep behavior disorder: from dreams to neurodegeneration. Neurobiol Dis 2012;46: 553-558.
29 Postuma RB, Lang AE, Massicotte-Marquez J, Montplaisir J: Potential early markers of Parkinson disease in idiopathic REM sleep behavior disorder. Neurology 2006;66:845851.

30 Schenck CH, Boeve BF, Mahowald MW: Delayed emergence of a parkinsonian disorder or dementia in $81 \%$ of older men initially diagnosed with idiopathic rapid eye movement sleep behavior disorder: a 16-year update on a previously reported series. Sleep Med 2013; 14:744-748.

1 Doty RL: Olfaction in Parkinson's disease and related disorders. Neurobiol Dis 2012;46: 527-552.
32 Stern Y: Cognitive reserve in ageing and $\mathrm{Al}$ zheimer's disease. Lancet Neurol 2012;11: 1006-1012.

33 Jankovic J, McDermott M, Carter J, Gauthier S, Goetz C, Golbe L, et al: Variable expression of Parkinson's disease: a base-line analysis of the DATATOP cohort. The Parkinson study group. Neurology 1990;40: 1529-1534.

34 van de Berg WD, Hepp DH, Dijkstra AA, Rozemuller JA, Berendse HW, Foncke E: Patterns of $\alpha$-synuclein pathology in incidental cases and clinical subtypes of Parkinson's disease. Parkinsonism Relat Disord 2012; 18(suppl 1):S28-S30. 